\title{
Growing up with your software
}

Stephen Wolfram, the designer of Mathematica, the computationalist's software package, hopes to get back to physics soon. But will the bug let him go free?

STEPHEN Wolfram, the enfant terrible turned software salesman, is looking forward to a brief sabbatical, which he plans to use for writing a book on some aspect of complex systems. He reckons that he will be able adequately to keep in touch with his business, Wolfram Research Inc., which is based in Illinois. Even if he should decide to spend his time off in California, he reckons that electronic mail should serve as well from there as it does now from wherever else he is.

Wolfram is, of course, a phenomenon. I first heard about him more than a decade ago from W. H. ("Willie") Fowler, who was boasting over lunch one day that Caltech (otherwise the California Institute of Technology) had just acquired from Britain a prodigy who had taken his $\mathrm{PhD}$ in just over a year. The truth is even more startling. Wolfram had abandoned his undergraduate physics course at Oxford after a year and a bit, and signed up instead as a graduate student at Pasadena. When he graduated in 1988 , he had just turned twenty. He had been hoping to become the only teenager of his acquaintance with a $\mathrm{PhD}$, but he was beaten by a few months.

That was in 1979. Last week, Wolfram was in London briefly after touching base in twelve European countries in fourteen days. He was off to Cambridge later in the evening, and then to the Soviet Union. The objective? To do propaganda for Mathematica, his own software package whose power, he claims, now extends beyond that of being a computer program capable of handling symbolic algebra to being a computer language in its own right. It can also produce the square root of $\pi$ to, say, 40 decimal places as quickly as if it were retrieving a predefined variable from immediate memory.

Wolfram has always been a computer buff. I first met him a few years after I first heard about him, when he had been exiled (if Princeton will forgive the term) to the Institute of Advanced Study. What had happened was that Wolfram had developed a computer program for calculating Feynmann integrals and had then broken the academic rules by founding a corporation to sell the product to his fellow academics.

His obsession at Princeton was the cellular automaton - von Neumann's conceptual device for representing a complex dynamical system by an array of simple microscopic elements (usually two-valued switches that are either ON or OFF, whose evolution in the course of time is determined by simple rules determining their interaction with other elements in the array. The serious objective was that of solving unsolved problems in fluid dynamics, but Wolfram had built an impressive collection of sea animal shells whose markings seemed evocative of the orderly patterns that could be induced to emerge from the chaotic behaviour of an array of cellular automata.

His toy was a machine the like of which I had not then seen elsewhere - a desktop computer with 1 megabyte of immediate memory. The need was plain -1 megabyte would be just enough to store a single image of high-definition screen. With the hardware ahead of the software, he had written the operating system himself. There were some hundreds of megabytes on hard disks in a separate box on which successive images would be stored. Sensing my envy of the toy, Wolfram advised me to buy stock in its manufacturer, now well-known as Sun Microsystems. As the world now knows, it was good advice. Wolfram is not to blame that I did not take it.

Now, at what must be all of thirty-three, Wolfram has not changed much. He was stocky ten years ago, and may be a little more so now. His hair has receded a little, making his forehead seem even broader, but is no tidier. He jeered politely about the restaurant, where neckties are required, but was not wearing sneakers. He remains buoyant with enthusiasm, and for Mathematica in particular, although he is also proud of the all electronic journal Complex systems, which seems to have been the first to give its mailing address as a Bitnet number and its "Instructions to authors" as a series of instructions for the typesetting language $T_{E} X$.

Mathematica is meant as a way of doing mathematics by machine that has its roots in Wolfram's automation of cellular automata nearly a decade ago. It seems also to be a product of Wolfram's own belief that, at least if you are he, the only way to come by a satisfactory program is to design and build it yourself. One might feel the same about one's motor-car, but it needs very little forethought to conclude that a person who insisted on building his own machines would have very little time left for using them. That is why Wolfram has become a business.

By his own account, it is also a successful business. "I have made a lot of money." There are no outside shareholders - only himself and his employees (who number about sixty). He is fiercely proud of the structure of his business, saying that he learned from his earlier venture that there is no point hiring a bunch of accountants and administrators to run the shell of a company within which the innovator will be coddled. Instead, you must begin by doing everything yourself, handing over parcels of work that can be successfully delegated.

But can a person so closely identified personally with a computer program delegate to others responsibility for its future? Wolfram is forgiveably ambiguous on this point. Users are always suggesting elaboration (although not as often as he had expected). He can, he says, leave it to others whom he trusts to add extra features to the system, but is less willing to delegate responsibility for new developments that are likely to affect the design of the system. And the crucial elements of the design are those that touch the interface between the program and its users, which determine how conveniently it can be used. One guesses that Wolfram will be worrying about the shape of Mathematica for, to a first approximation, the rest of time.

The users of Mathematica (more than 100,000 of them are registered, which means that they have paid for their copies) are scattered about the world. About a quarter of them are engineers, which is not surprising. For are not engineers the heaviest users of mathematics and at the same time the users least interested in the mathematics as such? But Wolfram is also delighted that the program has turned out to be a splendid way of generating educational materials as in the teaching of elementary calculus. His ambition for it seems to be that of a high-level shell that will support, as they say in the trade, any useful application its users may wish for.

Where does this leave Wolfram as a scientist? The next step is to be the book on complex systems. He also believes the epistemology of quantum mechanics to be ripe for sorting out. The grand problems of the Universe are by comparison less interesting. But, of course, there is also always the business of what will happen to computers and their capabilities. He is not interested in having Mathematica made capable of proving Euclid's theorems, but believes it has the potential to do thinking of a kind.

Wolfram carries Mathematica about with him, on a portable Compaq with 10 megabytes of immediate memory and a hard disk. He startles the waiters in the necktie restaurant by opening it on the linen tablecloth to show its tricks. "Look, here are the first forty digits of the square root of $\pi$, now ask for something else." So we tried a few integrals and solved the odd quadratic equation. $\mathrm{He}$ set up a cellular automaton, but it turned out to be a boring one - no chaos and so no order out of chaos. The toy now is not the machine, but the program. It will be interesting to see whether he can leave it alone during his sabbatical.

John Maddox 